\title{
Encuesta on-line de experiencias anómalo/ paranormales y su impacto emocional: Relación con género, edad y otras variables*
}

\author{
Alejandro Parra \\ Instituto de Psicología Paranormal
}

Recibido: 12 de diciembre del 2010 / Aprobado: 5 de marzo del 2011

Se diseñó una encuesta on-line dirigida a interesados en temas new age, esoterismo y simpatizantes de una diversidad de experiencias psicológicas anómalas, espirituales, y paranormales $(N=1733 ; 70 \%$ mujeres y $30 \%$ varones, Media edad = 31,31). Los obj etivos de este estudio fueron: 1) evaluar la frecuencia y porcentaje de catorce tipos de experiencias, 2) medir el grado de su impacto emocional, y 3) relacionar cada experiencia con variables demográficas, tales como género, edad, estado civil, orden de nacimiento, religión y espiritualidad. Las experiencias anómalo/paranormales / género / edad

On-line survey of anomalous/paranormal experiencies and their emotional impact: relationship with gender, age, and other variables The purposes of this study are to (1) evaluate la frequency and percentage of fourteen types of experiences; (2) measure the degree of emotional impact; and (3) relate each experience to demographic variables such as gender, age, marital status, birth order, religion and spirituality. To this end an online survey was designed to participants who were interested in topics such as "new age", esotericism and a range of anomalous, spiritual and paranormal psychological experiences $(N=1733 ; 70 \%$ female, 30\% males; median age $=31,31)$. Results show that the most frequent experiences were telepathy, premonitory dreams, sensations of a presence, and déjà-vu. Results also show that the most disturbing experiences were related to feelings of possessions, negative hallucinations and spontaneous psychokinesis.

anomalous/paranormal experiences / gender / age

\footnotetext{
* Deseamos expresar nuestra gratitud a la Fundación BIAL por su apoyo financiero durante el proceso de la presente investigación.

Correo electrónico: rapp@fibertel.com.ar
} 


\section{INTRODUCCIÓN}

Una experiencia paranormal puede ser definida como el conjunto de experiencias anómalas que sugieren la hipótesis de un tipo de interacción entre los organismos y su medio ambiente que parece contradecir el constructo espacio-temporal y energético que sostiene el paradigma científico moderno. Algunos autores (Irwin \& Watt, 2007; Cardeña, Krippner \& Lynn, 2000; Parra, 2003) han examinado estas experiencias paranormales en términos de la comunicación directa "mente a mente" -telepatía-, el conocimiento de eventos a distancia-visión remota o clarividencia-, información acerca del futuro o del pasado -precognición y psicometría, respectivamente- y la influencia mental directa sobre el entorno -influencia mental remota-.

Se ha explorado un amplio espectro de experiencias anómalas, las cuales parecen presentar mayor incidencia y frecuencia en la población general y se ha tratado de determinar qué características demográficas, psicológicas y fenomenológicas están implicadas (Irwin \& Watt, 2007; Rush, 1986; Stokes, 1997; Watt, 1994). Sobre dos muestras de 268 estudiantes y 354 habitantes adultos residentes en Charlottesville, Palmer (1979) llevó a cabo una encuesta postal sobre experiencias anómalo/paranormales. Relatos acerca de experiencias fuera del cuerpo, experiencias cercanas a la muerte, ver el aura, recuerdo de vidas pasadas, comunicación con los muertos fueron bastante frecuentes: más de la mitad dijo haber tenido al menos una experiencia; de hecho, muchos indicaron que tales experiencias habían afectado sus actitudes hacia la vida o estilos de vida.

Kohr (1980) usó el mismo cuestionario sobre una muestra de 406 miembros de la Association for Research and Enlightment, un centro de estudios relacionados con el desarrollo espiritual. Kohr obtuvo resultados similares al estudio de Palmer, y la tendencia a reportar un tipo particular de experiencia y a tener un alto número de experiencias anómalo/paranormales. Thalbourne encuestó a 402 miembros de la Society for Psychical Research y también encontró una alta incidencia de creencias y experiencias paranormales, con un puntaje promedio significativo en la Australian Sheep-Goat Scale. Alvarado y Zingrone (1999) examinaron un tipo de experiencia anómalo/paranormal particular sobre una muestra de lectores de una revista española especializada en temas new age, y encontraron que el $82 \%$ indicó haber tenido al menos una experiencia fuera del cuerpo, entre otras.

En un estudio anterior administramos dos encuestas de experiencias anómalo/paranormales sobre una población de personas practicantes de terapias alternativas y creencias new age. En un primer estudio ( $\mathrm{N}=432$; Gómez Montanelli y Parra, 2002) se obtuvo no solo tipo y frecuencia de experien- 
cias, sino si estas resultaron -de algún modo- perturbadoras y en qué grado, si consultaron por sus experiencias, quiénes fueron sus consultores, si iniciaron un tratamiento o buscaron consejo, y si su tratamiento por estas ayudó a disminuir su ansiedad. Más de los dos terceras partes reportó haber experimenado telepatía, sueños premonitorios, experiencias fuera del cuerpo, sanación psíquica y sensación de presencia. Encontramos que casi las dos terceras partes indicaron que al menos una de sus experiencias había resultado perturbadora. Aunque las dos terceras partes señalaron nunca haber consultado; quienes lo hicieron, sin embargo, señalaron que los parientes y amigos fueron las personas más consultadas, y la mitad manifestó que la respuesta de estas personas disminuyó la ansiedad.

La representatividad en las encuestas on-line refiere a la población de usuarios de internet en su totalidad (navegantes), aunque puede extenderse también a grupos específicos de usuarios de internet, usuarios de determinados websites, y grupos de usuarios que puedan acceder mediante algún tipo de dispositivo a la red. La representatividad de otros grupos poblacionales es posible cuando el acceso a internet en esos grupos esté lo suficientemente avanzado y dicho grado de desarrollo pueda demostrarse empíricamente (similar al del grado de penetración del teléfono en los hogares) (Mehrwer Durch Qualitat, 2001).
Hay dos ejemplos recientes de su uso en la literatura. Espinoza Paul (2004) diseñó una encuesta on-line de 46 preguntas, incluyendo experiencias aparicionales, variables demográficas (edad, sexo, estado civil, educación, religión y religiosidad), experiencias anómalo/paranormales, y absorción psicológica. De una muestra de 125 participantes peruanos de ambos sexos, la mayoría eran mujeres $(54 \%)$, solteros $(69 \%)$, universitarios $(70 \%)$, católicos $(75 \%)$ y moderadamente religiosos (38\%). El análisis de sus resultados reveló que el 55\% (N =69) de los encuestados reportó haber tenido alguna vez en su vida una experiencia aparicional. Parra (2009) obtuvo datos de otra encuesta on-line de experiencias de telepatía (telefónica) sobre una muestra que comprende 372 participantes, que incluyó a individuos residentes en Buenos Aires y Gran Buenos Aires. La experiencia de ser "emisor" telepático en términos de pensar en la persona-objetivo al momento de llamar por teléfono fue reportada por un gran número de participantes (96\%), en tanto que la experiencia de ser "receptor" en términos de saber de algún modo quién era la persona-objetivo al momento de sonar el teléfono, fue reportada por un igualmente significativo porcentaje de personas, aunque menor que como receptor $(82 \%)$.

El presente estudio es una investigación exploratoria que intenta replicar un resultado previo (Gómez Montanelli \& Parra, 2002) con una muestra mayor de 
individuos reclutados mediante una encuesta on-line, cuyos objetivos son: 1) evaluar catorce experiencias anómalo/ paranormales y sus frecuencias, 2) medir el grado de impacto emocional de cada experiencia, y 3) relacionar la frecuencia de cada experiencia con variables demográficas, tales como género, edad, estado civil, orden de nacimiento, religión, y espiritualidad.

\section{MÉTOdo}

\section{Participantes}

La muestra comprende 1733 participantes, de ambos sexos 1206 mujeres (70\%) y 527 varones (30\%), rango etario de 13 a 85 años (Media $=31,31 ; \mathrm{SD}=11.86$; Media varones $=29,46 ; \mathrm{SD}=11,53$; Media mujeres $=32,12, \mathrm{SD}=11,92$ ), que incluyó a individuos (véase http:// www.alipsi.com.ar/encuesta_telepatia. asp) de varios países hispanoparlantes (66\%), principalmente Argentina (52\%), España (10\%) y México (4\%). De las principales características demográficas reportadas, el 58\% indicó ser soltero, el $25 \%$ profesionales, el $40 \%$ moderadamente espiritual y el $41 \%$ tener pensamiento politico liberal.

\section{Instrumentos}

Se desarrolló un inventario autoadministrable para reunir información sobre catorce experiencias anómalo/paranor- males espontáneas (encuesta de experiencias paranormales), que incluyen sueños premonitorios, telepatía, ver el aura, experiencia fuera del cuerpo, experiencia cercana a la muerte, recuerdo de vidas pasadas, sensación de presencia, posesión espiritual, alucinación negativa, ${ }^{1}$ psicokinesis espontánea, sanación psíquica, déjà-vú, experiencias místicas y experiencias aparicionales. Si la respuesta es "sí", cada pregunta también evalúa la frecuencia de la experiencia (una vez, a veces, frecuentemente), y su impacto emocional (siendo $1=$ positiva $\mathrm{y}$ enriquecedora $\mathrm{y} 7=$ negativa y perturbadora).

Las instrucciones para completar el cuestionario fueron:

Las siguientes preguntas se refieren a diferentes tipos de experiencia que pudieron haberle ocurrido. Por favor, lea cada pregunta y conteste tildando con su mouse la frecuencia de esta experiencia (una vez, algunas veces, casi siempre) según corresponda para usted. No hay respuestas buenas o malas; todas sirven. Tampoco hay preguntas de doble intención. Si in-

1 La alucinación "negativa" es una falla en la percepción de un objeto, a diferencia de la alucinación, en la cual el individuo ve algo o escucha una voz cuya presencia no es real. En otras palabras, el objeto está realmente presente pero el individuo no tiene consciencia de este (véase Parra, 2008a). A menudo se atribuyen interpretaciones paranormales a esta experiencia perceptual anómala. 
dicó NUNCA, continúe con el resto de las preguntas. Finalmente, indique si cada experiencia ha sido positiva o ha sido negativa; Positiva significa que la experiencia ha resultado enriquecedora, agradable o placentera; Negativa significa que la experiencia ha resultado emocionalmente perturbadora o displacentera. Un puntaje cercano a 4 indica que no ha resultado ni displacentera ni placentera.

\section{Procedimiento}

La versión digital de la encuesta fue diseñada por Alejandro Jarandilla, de Publiland, e incluyó individuos que completaron totalmente el cuestionario (versión htm) en el sitio web del Instituto de Psicología Paranormal (véase http:// www.alipsi.com.ar/encuesta_telepatia. asp). La muestra de 1733 participantes fue colectada entre el 13 de febrero del 2004 y el 13 de diciembre del 2009. Las encuestas se imprimieron en papel y se ingresaron manualmente a una matriz diseñada mediante el programa de análisis estadístico (SPSS v.18).

\section{Análisis}

El test Kolmogorov-Smirnov mostró una distribución asimétrica $(\mathrm{p}<.001$, rango $=.23$ a .51 , Media $=.32$ para las catorce experiencias), por lo cual se decidió aplicar estadística no-paramétrica ( $U$ de Mann-Whitney y Rho de Spearman) para el análisis de los datos.

\section{Resultados}

Se correlacionó la medida de impacto emocional de la experiencia con su respectiva experiencia anómalo/paranormal. Los resultados muestran que solo cuatro de las catorce experiencias (28\%) mostraron significación estadística con su impacto emocional. Por ejemplo, Ver el aura, Experiencia fuera del cuerpo y Sanación psíquica mostraron que a mayor frecuencia de experiencias, el impacto emocional es más positivo; en cambio, Alucinación negativa mostró la relación inversa (a mayor frecuencia mayor perturbación emocional). El resto de las experiencias no mostró significación alguna (véase la tabla 3 ).

De manera similar a un estudio de Zingrone y Alvarado (1999), se generó un valor llamado "Index" para medir el número de diferentes tipos de experiencias por sujeto (Rango $=1$ a 14; Media $=9 ; \mathrm{SD}=2,65)$ y se empleó este valor para correlacionar y comparar otras variables demográficas, como Estado civil, Espiritualidad, Religión y Orden de nacimiento. 
Tabla 1

Tipo y frecuencia de las experiencias anómalo/paranormales*

\begin{tabular}{|c|c|c|c|c|c|c|c|c|c|c|}
\hline \multirow[b]{2}{*}{ Experiencias } & \multicolumn{2}{|c|}{1} & \multicolumn{2}{|c|}{2} & \multicolumn{2}{|c|}{3} & \multicolumn{2}{|c|}{ sí } & \multicolumn{2}{|c|}{ NO } \\
\hline & $\mathrm{N}$ & $\%$ & $\mathrm{~N}$ & $\%$ & $\mathrm{~N}$ & $\%$ & $\mathbf{N}$ & $\%$ & $\mathbf{N}$ & $\%$ \\
\hline 1. Sueños premonitorios & 213 & 12.3 & 999 & 57.6 & 273 & 15.8 & 1485 & 85.7 & 248 & 14.3 \\
\hline 2. Telepatía & 195 & 11.3 & 986 & 56.9 & 300 & 17.3 & 1481 & 85.5 & 252 & 14.5 \\
\hline 3. Ver el aura & 215 & 12.4 & 481 & 27.8 & 187 & 10.8 & 883 & 51.0 & 850 & 49.0 \\
\hline $\begin{array}{l}\text { 4. Experiencia fuera del } \\
\text { cuerpo }\end{array}$ & 390 & 22.5 & 614 & 35.4 & 104 & 6.0 & 625 & 36.1 & 1108 & 63.9 \\
\hline $\begin{array}{l}\text { 5. Experiencia cercana a la } \\
\text { muerte }\end{array}$ & 167 & 6.4 & - & - & - & - & 167 & 6.4 & 1566 & 90.4 \\
\hline 6. Recuerdo de vida pasada & 219 & 12.6 & 745 & 43.0 & 244 & 14.1 & 1208 & 69.7 & 525 & 30.3 \\
\hline 7. Sensación de presencia & 103 & 5.9 & 903 & 52.1 & 622 & 35.9 & 1628 & 93.9 & 105 & 6.1 \\
\hline 8. Posesión espiritual & 305 & 17.6 & 441 & 25.4 & 65 & 3.8 & 811 & 46.8 & 922 & 53.2 \\
\hline 9. Alucinación negativa & 164 & 9.5 & 918 & 53.0 & 341 & 19.7 & 1423 & 82.1 & 310 & 17.9 \\
\hline 10. Psicokinesis espontánea & 247 & 14.3 & 638 & 36.8 & 142 & 8.2 & 1027 & 59.3 & 706 & 40.7 \\
\hline 11. Sanación & 232 & 13.4 & 546 & 31.5 & 164 & 9.5 & 942 & 54.4 & 791 & 45.6 \\
\hline 12. Déjà vu & 78 & 4.5 & 978 & 56.4 & 579 & 33.4 & 1635 & 94.3 & 98 & 5.7 \\
\hline 13. Experiencia mística & 226 & 13.0 & 412 & 23.8 & 144 & 8.3 & 782 & 45.1 & 951 & 54.9 \\
\hline 14. Apariciones & 233 & 13.4 & 524 & 30.2 & 185 & 10.7 & 942 & 54.4 & 791 & 45.6 \\
\hline
\end{tabular}

* 1 = Una vez. 2 = Algunas veces. 3 = Frecuentemente.

Tabla 2

Medias y desvío de impacto emocional de experiencias anómalo/paranormales*

\begin{tabular}{lrcc}
\hline Experiencias & N & Media & SD \\
\hline 1. Sueños premonitorios & 1485 & 3.41 & 1.78 \\
2. Telepatía & 1479 & 2.82 & 1.76 \\
3. Ver el aura & 883 & 2.72 & 1.76 \\
4. Experiencia fuera del cuerpo & 1108 & 3.37 & 1.96 \\
5. Experiencia cercana a la muerte & 166 & 3.35 & 2.11 \\
6. Recuerdo de vidas pasadas & 1208 & 3.15 & 1.72 \\
7. Sensación de presencia & 1628 & 3.98 & 1.83 \\
8. Posesión & 810 & 4.78 & 1.9 \\
9. Alucinación negativa & 1423 & 4.15 & 1.62 \\
10. Psicokinesis espontánea & 1026 & 4.08 & 1.69 \\
11. Sanación & 941 & 2.30 & 1.70 \\
12. Déjà vu & 1632 & 3.14 & 1.65 \\
13. Experiencia mística & 781 & 2.41 & 1.81 \\
14. Apariciones & 939 & 3.76 & 1.96 \\
\hline
\end{tabular}

* 1 = Positiva y enriquecedora a 7 = Negativa y perturbadora. 


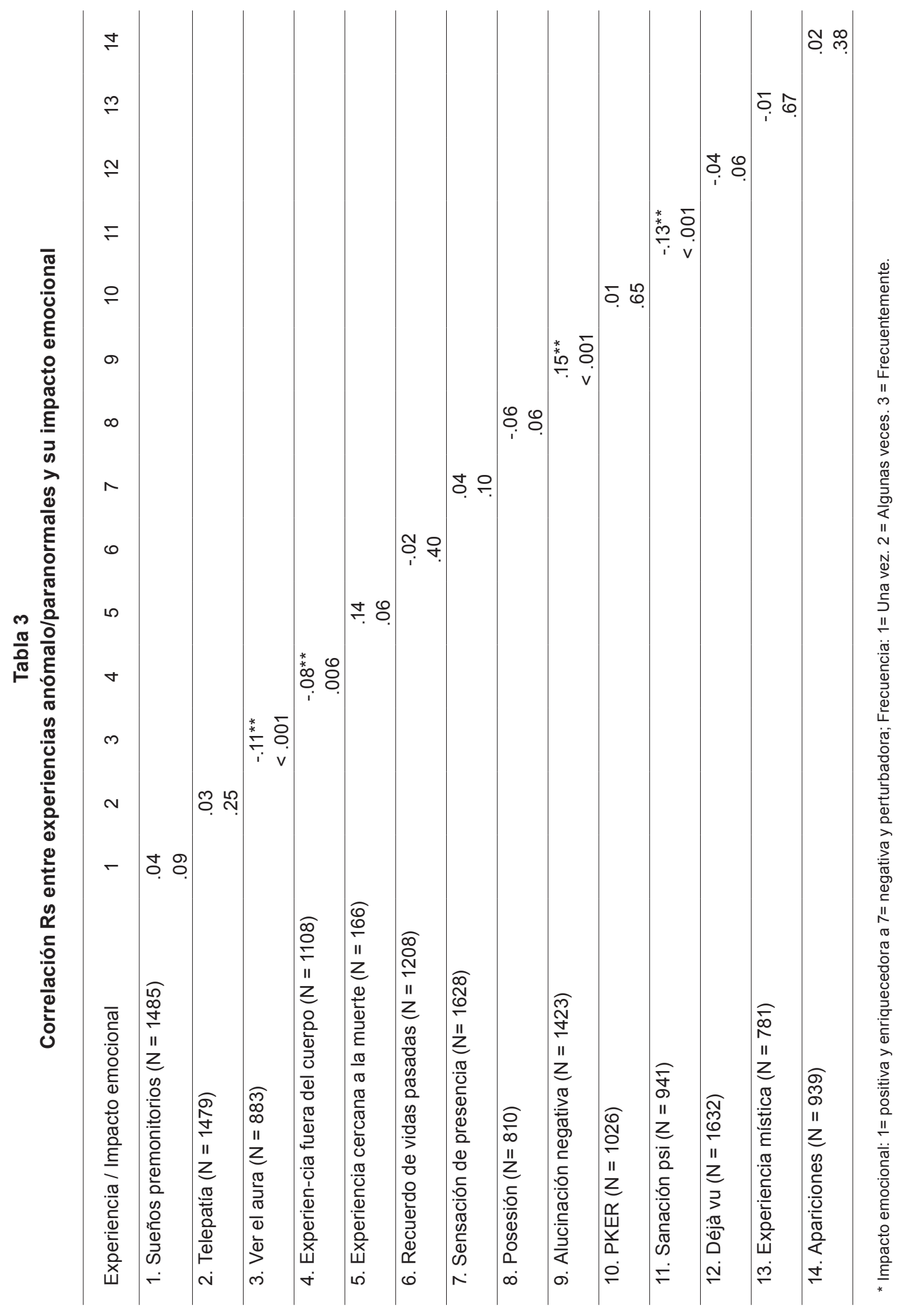


Tabla 4

Comparación de experiencias anómalo/paranormales entre varones y mujeres*

\begin{tabular}{|c|c|c|c|c|c|c|}
\hline $\begin{array}{l}\text { Experiencias } \\
\text { anómalo/paranormales }\end{array}$ & Género & Media & SD & $\begin{array}{l}\text { U de Mann- } \\
\text { Whittney }\end{array}$ & $\mathbf{z}$ & $\mathbf{p}$ \\
\hline 1. Sueños premonitorios & $\begin{array}{l}\text { mujer } \\
\text { varón }\end{array}$ & $\begin{array}{l}1.79 \\
1.65\end{array}$ & $\begin{array}{l}.87 \\
.91\end{array}$ & 291249.0 & -3.09 & .002 \\
\hline 2. Telepatía & $\begin{array}{l}\text { mujer } \\
\text { varón }\end{array}$ & $\begin{array}{l}1.83 \\
1.62\end{array}$ & $\begin{array}{l}.86 \\
.96\end{array}$ & 281807.0 & -4.18 & $<.001$ \\
\hline 3. Ver el aura & $\begin{array}{l}\text { mujer } \\
\text { varón }\end{array}$ & $\begin{array}{r}.97 \\
1.07\end{array}$ & $\begin{array}{l}1.06 \\
1.15\end{array}$ & 305449.5 & -1.39 & .16 \\
\hline 4. Experiencia fuera del cuerpo & $\begin{array}{l}\text { mujer } \\
\text { varón }\end{array}$ & $\begin{array}{l}1.11 \\
1.12\end{array}$ & $\begin{array}{l}.97 \\
.97\end{array}$ & 316503.0 & -.14 & .88 \\
\hline 5. Experiencia cercana a la muerte & $\begin{array}{l}\text { mujer } \\
\text { varón }\end{array}$ & $\begin{array}{l}.14 \\
.13\end{array}$ & $\begin{array}{l}.46 \\
.47\end{array}$ & 309100.5 & -1.77 & .07 \\
\hline 6. Recuerdo de vidas pasadas & $\begin{array}{l}\text { mujer } \\
\text { varón }\end{array}$ & $\begin{array}{l}1.44 \\
1.33\end{array}$ & $\begin{array}{l}1.05 \\
1.07\end{array}$ & 300754.0 & -1.886 & .05 \\
\hline 7. Sensación de presencia & $\begin{array}{l}\text { mujer } \\
\text { varón }\end{array}$ & $\begin{array}{l}2.22 \\
2.08\end{array}$ & $\begin{array}{l}.77 \\
.82\end{array}$ & 288459.5 & -3.396 & $<.001$ \\
\hline 8. Posesión & $\begin{array}{l}\text { mujer } \\
\text { varón }\end{array}$ & $\begin{array}{l}.79 \\
.80\end{array}$ & $\begin{array}{l}.94 \\
.96\end{array}$ & 317045.0 & -.084 & .93 \\
\hline 9. Alucinación "negativa" & $\begin{array}{l}\text { mujer } \\
\text { varón }\end{array}$ & $\begin{array}{l}1.83 \\
1.54\end{array}$ & $\begin{array}{r}.92 \\
1.03\end{array}$ & 269361.0 & -5.52 & $<.001$ \\
\hline 10. Psicokinesis espontánea & $\begin{array}{l}\text { mujer } \\
\text { varón }\end{array}$ & $\begin{array}{l}1.16 \\
1.06\end{array}$ & $\begin{array}{l}1.04 \\
1.04\end{array}$ & 301021.5 & -1.86 & .06 \\
\hline 11. Sanación & $\begin{array}{l}\text { mujer } \\
\text { varón }\end{array}$ & $\begin{array}{l}1.02 \\
1.10\end{array}$ & $\begin{array}{l}1.07 \\
1.06\end{array}$ & 304339.0 & -1.50 & .13 \\
\hline 12. Déjà vu & $\begin{array}{l}\text { mujer } \\
\text { varón }\end{array}$ & $\begin{array}{l}2.21 \\
2.11\end{array}$ & $\begin{array}{l}.70 \\
.85\end{array}$ & 306617.0 & -1.31 & .18 \\
\hline 13. Experiencia mística & $\begin{array}{l}\text { mujer } \\
\text { varón }\end{array}$ & $\begin{array}{l}.84 \\
.90\end{array}$ & $\begin{array}{l}1.03 \\
1.07\end{array}$ & 307553.0 & -1.18 & .23 \\
\hline 14. Ver apariciones & $\begin{array}{l}\text { mujer } \\
\text { varón }\end{array}$ & $\begin{array}{r}1.09 \\
.99\end{array}$ & $\begin{array}{l}1.08 \\
1.09\end{array}$ & 300953.5 & -1.87 & .06 \\
\hline Index & $\begin{array}{l}\text { mujer } \\
\text { varón }\end{array}$ & $\begin{array}{l}9.06 \\
8.67\end{array}$ & $\begin{array}{l}2.594 \\
2.780\end{array}$ & 293656.5 & -2.53 & .011 \\
\hline
\end{tabular}

* Mujeres $\mathrm{N}=1206$; Varones $\mathrm{N}=527$. 
- Género.- Se encontraron diferencias de género en experiencias tales como Sueños premonitorios (Mujeres $\mathrm{M}=1.79$ vs. Varones $1.65, \mathrm{p}<$ .002 ), Telepatía (Mujeres $\mathrm{M}=1.83$ vs. Varones $1.62, \mathrm{p}<.001)$, Sensación de presencia (Mujeres $\mathrm{M}=2.22$ vs. Varones $2.08, \mathrm{p}<.001)$, Alucinación negativa (Mujeres $\mathrm{M}=1.83$ vs. Varones 1.54, $\mathrm{p}<.001)$, y Apariciones (Mujeres $\mathrm{M}=1.09$ vs. Varones $.99, \mathrm{p}<.001)$. Para el resto de las experiencias, las mujeres tendieron a puntuar más alto (aunque no significativamente) en comparación con los varones (véase la tabla 4).

- Edad.- Algunas experiencias correlacionaron positiva y significativamente en edad, por ejemplo, Telepa- tía $(\mathrm{R} s=.17, \mathrm{p}<.001)$, Experiencias cercanas a la muerte $(\mathrm{R} s=.08, \mathrm{p}<$ .001), Sanación psíquica ( $\mathrm{R} s=.20, \mathrm{p}$ $<.001)$ y Experiencia mística $(\mathrm{R} s=$ $.16, \mathrm{p}<.001)$. En dirección inversa, otras experiencias se presentan en individuos más jóvenes; por ejemplo, Sensación de presencia $(\mathrm{R} s=$ $.15, \mathrm{p}<.001$ ), Posesión espiritual $(\mathrm{R} s=.07, \mathrm{p}=.003)$, y Déjà-vu $(\mathrm{R} s=$ $.18, \mathrm{p}=.003$ ) (véase la tabla 5).

- Espiritualidad.- Se correlacionó la intensidad de la espiritualidad con la frecuencia de las experiencias anómalo/paranormales. Todas las correlaciones fueron positivas y significativas (Media $\mathrm{R} s=.24$ ), con un valor de $\mathrm{p}<.001$ (véase la tabla 6 ).

Tabla 5

Correlación entre edad y experiencias anómalo/paranormales*

\begin{tabular}{lccccccccccccccc}
\hline Experiencia & $\mathbf{1}$ & $\mathbf{2}$ & $\mathbf{3}$ & $\mathbf{4}$ & $\mathbf{5}$ & $\mathbf{6}$ & $\mathbf{7}$ & $\mathbf{8}$ & $\mathbf{9}$ & $\mathbf{1 0}$ & $\mathbf{1 1}$ & $\mathbf{1 2}$ & $\mathbf{1 3}$ & $\mathbf{1 4}$ & Index \\
\hline Edad Rs & -.008 & $.17^{* *}$ & .001 & .04 & $.08^{* *}$ & .030 & $-.15^{* *}$ & $-.07^{* *}$ & $-.08^{* *}$ & .034 & $.20^{* *}$ & $-.18^{* *}$ & $.16^{* *}$ & -.01 & .05 \\
$\mathrm{p}$ & .739 & $<.001$ & .982 & .061 & $<.001$ & .215 & $<.001$ & .003 & $<.001$ & .158 & $<.001<.001<.001$ & .622 & .02 \\
\hline
\end{tabular}

${ }^{*}$ Min. $=13$ a Max. $=83$ años.

Tabla 6

Correlación entre experiencias anómalo/paranormales y espiritualidad*

\begin{tabular}{lccccccccccccccc}
\hline Experiencia & $\mathbf{1}$ & $\mathbf{2}$ & $\mathbf{3}$ & $\mathbf{4}$ & $\mathbf{5}$ & $\mathbf{6}$ & $\mathbf{7}$ & $\mathbf{8}$ & $\mathbf{9}$ & $\mathbf{1 0}$ & $\mathbf{1 1}$ & 12 & 13 & 14 & Index \\
\hline Espiritual RS & $.08^{* *}$ & $.22^{* *}$ & $.16^{* *}$ & $.12^{* *}$ & $.06^{*}$ & $.09^{* *}$ & $.10^{* *}$ & $.08^{* *}$ & $.06^{* *}$ & $.12^{* *}$ & $.21^{* *}$ & $.04^{*}$ & $.32^{* *}$ & $.13^{* *}$ & $.24^{* *}$ \\
$\mathrm{p}$ & .001 & $<.001<.001<.001$ & .01 & $<.001<.001<.001$ & .005 & $<.001<.001$ & .04 & $<.001<.001<.001$ \\
\hline
\end{tabular}

* $1=$ Soy poco espiritual a $5=$ Soy muy espiritual. 
- Impacto emocional.- Se comparó el impacto emocional de las experiencias anómalo/paranormales entre varones y mujeres, pero se encontraron diferencias marginalmente significativas solo en cuatro (18\%) de las catorce experiencias. En términos generales, los varones tendieron a mostrar mayor impacto emocional negativo en comparación con las mujeres en Telepatía, ECM y Psicokinesis espontánea (excepto Posesión, la cual se mostró más negativa en mujeres que en varones) (véase la tabla 7).

\section{Tabla 7}

Comparación del impacto emocional de las experiencias entre varones y mujeres

\begin{tabular}{|c|c|c|c|c|c|c|c|}
\hline $\begin{array}{l}\text { Experiencias } \\
\text { anómalo/paranormales }\end{array}$ & Género & $\mathbf{N}$ & Media & SD & $\begin{array}{l}\text { U de Mann- } \\
\text { Whittney }\end{array}$ & $\mathbf{z}$ & $\mathbf{p}$ \\
\hline 1. Sueños premonitorios & $\begin{array}{l}\text { mujer } \\
\text { varón }\end{array}$ & $\begin{array}{r}1050 \\
435 \\
\end{array}$ & $\begin{array}{l}3.45 \\
3.33 \\
\end{array}$ & $\begin{array}{l}1.77 \\
1.79\end{array}$ & 219368.0 & -1.22 & .221 \\
\hline 2. Telepatía & $\begin{array}{l}\text { mujer } \\
\text { varón }\end{array}$ & $\begin{array}{r}1056 \\
423 \\
\end{array}$ & $\begin{array}{l}2.74 \\
3.02 \\
\end{array}$ & $\begin{array}{l}1.70 \\
1.88 \\
\end{array}$ & 206779.0 & -2.29 & .02 \\
\hline 3. Ver el aura & $\begin{array}{l}\text { mujer } \\
\text { varón }\end{array}$ & $\begin{array}{l}611 \\
272 \\
\end{array}$ & $\begin{array}{l}2.65 \\
2.89\end{array}$ & $\begin{array}{l}1.69 \\
1.89\end{array}$ & 78309.0 & -1.41 & .15 \\
\hline 4. Experiencia fuera del cuerpo & $\begin{array}{l}\text { mujer } \\
\text { varón }\end{array}$ & $\begin{array}{l}772 \\
336 \\
\end{array}$ & $\begin{array}{l}3.39 \\
3.32 \\
\end{array}$ & $\begin{array}{l}1.93 \\
2.01\end{array}$ & 126252.5 & -.71 & .47 \\
\hline 5. Experiencia cercana a la muerte & $\begin{array}{l}\text { mujer } \\
\text { varón }\end{array}$ & $\begin{array}{r}126 \\
40 \\
\end{array}$ & $\begin{array}{l}3.13 \\
4.03 \\
\end{array}$ & $\begin{array}{l}2.01 \\
2.29\end{array}$ & 1967.5 & -2.13 & .03 \\
\hline 6. Recuerdo de vidas pasadas & $\begin{array}{l}\text { mujer } \\
\text { varón }\end{array}$ & $\begin{array}{l}859 \\
349\end{array}$ & $\begin{array}{l}3.14 \\
3.18 \\
\end{array}$ & $\begin{array}{l}1.68 \\
1.82 \\
\end{array}$ & 149630.5 & -.04 & .96 \\
\hline 7. Sensación de presencia & $\begin{array}{l}\text { mujer } \\
\text { varón }\end{array}$ & $\begin{array}{r}1142 \\
486 \\
\end{array}$ & $\begin{array}{l}3.95 \\
4.05\end{array}$ & $\begin{array}{l}1.82 \\
1.88\end{array}$ & 268793.0 & -1.01 & .30 \\
\hline 8. Posesión & $\begin{array}{l}\text { mujer } \\
\text { varón }\end{array}$ & $\begin{array}{l}565 \\
245 \\
\end{array}$ & $\begin{array}{l}4.88 \\
4.56 \\
\end{array}$ & $\begin{array}{l}1.92 \\
1.99 \\
\end{array}$ & 62800.5 & -2.13 & .03 \\
\hline 9. Alucinación negativa & $\begin{array}{l}\text { mujer } \\
\text { varón }\end{array}$ & $\begin{array}{r}1025 \\
398 \\
\end{array}$ & $\begin{array}{l}4.17 \\
4.11 \\
\end{array}$ & $\begin{array}{l}1.62 \\
1.62 \\
\end{array}$ & 200129.00 & -.56 & .57 \\
\hline 10. Psicokinesis espontánea & $\begin{array}{l}\text { mujer } \\
\text { varón }\end{array}$ & $\begin{array}{l}731 \\
295 \\
\end{array}$ & $\begin{array}{r}4.18 \\
3.84 \\
\end{array}$ & $\begin{array}{l}1.68 \\
1.72 \\
\end{array}$ & 96985.50 & -2.57 & .01 \\
\hline 11. Sanación & $\begin{array}{l}\text { mujer } \\
\text { varón }\end{array}$ & $\begin{array}{l}639 \\
302 \\
\end{array}$ & $\begin{array}{l}2.28 \\
2.34 \\
\end{array}$ & $\begin{array}{l}1.64 \\
1.80 \\
\end{array}$ & 96314.50 & -.04 & .96 \\
\hline 12. Déjà-vu & $\begin{array}{l}\text { mujer } \\
\text { varón }\end{array}$ & $\begin{array}{r}1154 \\
478 \\
\end{array}$ & $\begin{array}{l}3.18 \\
3.04 \\
\end{array}$ & $\begin{array}{l}1.63 \\
1.70 \\
\end{array}$ & 260660.0 & -1.78 & .07 \\
\hline 13. Experiencia mística & $\begin{array}{l}\text { mujer } \\
\text { varón }\end{array}$ & $\begin{array}{l}532 \\
249 \\
\end{array}$ & $\begin{array}{l}2.35 \\
2.55 \\
\end{array}$ & $\begin{array}{l}1.78 \\
1.88 \\
\end{array}$ & 62303.0 & -1.43 & .15 \\
\hline 14. Apariciones & $\begin{array}{l}\text { mujer } \\
\text { varón }\end{array}$ & $\begin{array}{l}673 \\
266 \\
\end{array}$ & $\begin{array}{l}3.80 \\
3.66 \\
\end{array}$ & $\begin{array}{l}1.92 \\
2.03\end{array}$ & 85600.0 & -1.05 & .29 \\
\hline
\end{tabular}


Tabla 8

Correlación entre impacto emocional de las experiencias y espiritualidad*

\begin{tabular}{lccccccccccccccc}
\hline & $\mathbf{1}$ & $\mathbf{2}$ & $\mathbf{3}$ & $\mathbf{4}$ & $\mathbf{5}$ & $\mathbf{6}$ & $\mathbf{7}$ & $\mathbf{8}$ & $\mathbf{9}$ & $\mathbf{1 0}$ & $\mathbf{1 1}$ & $\mathbf{1 2}$ & $\mathbf{1 3}$ & $\mathbf{1 4}$ & Index \\
\hline $\mathrm{N}$ & 1485 & 1479 & 883 & 1108 & 166 & 1208 & 1628 & 810 & 1423 & 1026 & 941 & 1632 & 781 & 939 & 1733 \\
\hline $\begin{array}{l}\text { Espirituali- } \\
\text { dad }^{* *}(\mathrm{Rs})\end{array}$ & $-.13^{* *}$ & $-.14^{* *}$ & $-.08^{* *}$ & $-.15^{* *}$ & -.04 & $-.12^{* *}$ & $-.14^{* *}$ & -.05 & $-.09^{* *}$ & $-.09^{* *}$ & $-.21^{* *}$ & $-.14^{* *}$ & $-.13^{* *}$ & $-.14^{* *}$ & $.24^{* *}$ \\
\hline $\mathrm{p}$ & $<.001<.001<.01$ & $<.001$ & .54 & $<.001<.001$ & .10 & $<.001$ & .002 & $<.001<.001<.001<.001<.001$ \\
\hline
\end{tabular}

* Impacto emocional: 1 = Muy positiva y benéfica a 7 = Muy negativa y perturbadora.

** Espiritualidad: 1 = No soy espiritual a 5 = Soy extremadamente espiritual.

- Espiritualidad.- Se correlacionó la intensidad de la espiritualidad con el impacto emocional de la experiencia. Doce de las catorce correlaciones $(85 \%)$ fueron significativas, siendo Sanación la más robusta (Rs $=.21, \mathrm{p}<.001)$.

\section{DISCUSIÓN}

En este estudio las experiencias anómalo/paranormales que se presentan con mayor frecuencia son Déjà-vu, Sensación de presencia, Sueños premonitorios y Telepatía; la experiencia con menor frecuencia es Experiencias cercanas a la muerte. Las experiencias emocionalmente más positivas son Sanación psi y Ver el aura, en cambio las más perturbadoras son Posesión, Alucinación negativa y Psicokinesis espontánea. De hecho, la experiencia de ver el aura, la experiencia fuera del cuerpo y la sanación mostraron que individuos que las reportan con frecuencia experimentan emociones más positivas, pero esto no ocurre con el resto de las experiencias.
Una de las ventajas de las encuestas on-line es el amplio espectro de casos que es posible coleccionar de una manera rápida, accesible y eficaz. Es importante notar, sin embargo, que la web solo proporciona: 1) la desventaja de atraer a poblaciones de individuos con intereses específicos, lo cual puede no ser representativa de la población general, 2) no es posible discriminar pseudoexperiencias (a menos que algunas personas amplíen cada respuesta exponiendo una narración de esta) para determinar la veridicabilidad de psi, y 3) discriminar cuestionarios con información distorsionada o exagerada.

Como ejemplo, se expone aquí una tabla que permite comparar los resultados del mismo cuestionario sobre una muestra de lectores de una revista popular de interés paranormal (Año/Cero) en la cual se publicó una encuesta empleando las mismas preguntas que el cuestionario del presente estudio (excepto Alucinación negativa, Déjà-vu y Experiencia mística): 
Tabla 9

Comparación entre ambos estudios

\begin{tabular}{lrr}
\hline Experiencias anómalo/paranormales & Estudio2002* & Estudio actual** \\
\hline 1. Sueños premonitorios & $304(70,3 \%)$ & $1485(85,7 \%)$ \\
2. Telepatía & $349(80,7 \%)$ & $1481(85,5 \%)$ \\
3. Ver el aura & $224(51,8 \%)$ & $883(51,0 \%)$ \\
4. Experiencia fuera del cuerpo & $309(28,5 \%)$ & $625(36,1 \%)$ \\
5. Experiencia cercana a la muerte & $60(13,8 \%)$ & $167(6,4 \%)$ \\
6. Recuerdo de vida pasada & $279(64,5 \%)$ & $1208(69,7 \%)$ \\
7. Sensación de presencia & $336(77,7 \%)$ & $1628(93,9 \%)$ \\
8. Posesión espiritual & $179(41,4 \%)$ & $811(46,8 \%)$ \\
9. Alucinación negativa & - & $1423(82,1 \%)$ \\
10. Psicokinesis espontánea & $219(50,6 \%)$ & $1027(59,3 \%)$ \\
11. Sanación & $228(52,7 \%)$ & $942(54,4 \%)$ \\
12. Déjà vu & - & $1635(94,3 \%)$ \\
13. Experiencia mística & - & $782(45,1 \%)$ \\
14. Apariciones & $111(25,6 \%)$ & $942(54,4 \%)$ \\
\hline
\end{tabular}

* Muestra argentina.

** Muestra argentina y de otros países hispanoparlantes.

- Relación entre variables demográficas y experiencias anómalo/paranormales.- Entre las variables demográficas incluidas en el cuestionario, por ejemplo, las mujeres tendieron a reportar un número relativamente más alto de experiencias paranormales en comparación con los varones, tales como Sueños premonitorios, Telepatía, Sensación de presencia, Alucinación negativa y Apariciones (hay una tendencia general, pero no significativa, a reportar más experiencias que los varones). También una tendencia a reportar más experiencias de Sensación de presencia, Posesión espiritual y Déjà-vu en individuos jóvenes, lo cual confirma estudios previos que examinaron muestras no probabilísticas (Parra, 2008a, 2008b, 2008c, 2008d; 2009a, 2009b; 2010), y en sentido análogo, una relación entre la espiritualidad y la frecuencia de experiencias, lo cual podría indicar que tales experiencias refuerzan de algún modo la espiritualidad (Parra, 2008d).

- Experiencias anómalo/paranormales, estado civil, religión y orden de nacimiento.- Es interesante notar que individuos con relaciones maritales rotas indicaron un número relativamente mayor de experiencias anómalo/paranormales, lo cual confirma estudios previos (Palmer, 1979; Thalbourne, 2001; Zingrone \& Alvarado, 1997). Practicantes de filosofías orientales (budismo, me- 
ditación, new age, etcétera), experimentaron mayor número de experiencias anómalo/paranormales $(\mathrm{N}=$ 79 , rango promedio $=1128,79 ; \mathrm{gl}=$ 5; Kruskal-Wallis test $\mathrm{p}<, 001$ ), lo cual también es consistente con espiritualidad $(\mathrm{N}=79$, rango promedio $=1066 ; \mathrm{gl}=5$; Kruskal-Wallis test $\mathrm{p}<$,001) (Palmer, 1979). Para Orden de nacimiento se encontró que "Hijo único" tiende a experimentar mayor número de experiencias anómalo/paranormales $(\mathrm{N}=203$, rango promedio $=913,45 ; \mathrm{gl}=3$; KruskalWallis test $p=, 004)$ en comparación con primero, segundo y último nacimiento.

- Relación entre impacto emocional de las experiencias y otras variables demográficas.- Solo en cuatro (28\%) de las catorce experiencias, los varones tendieron a mostrar más emociones negativas en comparación con las mujeres, así como una leve tendencia general en los varones en las experiencias, y doce (85\%) mostraron una tendencia a puntuar más alto en espiritualidad en términos de emociones positivas (en particular, Sanación).

\section{CONCLUSIONES}

El abordaje de las experiencias anómalo/paranormales debería ser un tema de interés por parte de los psicólogos clínicos. Las personas que han pasado por tales experiencias tienen dudas de aquello que les ha ocurrido, así como también el tipo de reacción o impacto emocional que han tenido. Esta es un área parcialmente descuidada en parapsicología a pesar del rol que las emociones juegan en los procesos cognitivos y procesamiento de información.

Futuros estudios deberían determinar, mediante entrevistas u otras escalas, qué tipo de emociones negativas o positivas específicas emergen de unas $u$ otras experiencias anómalo/paranormales. Además, tales experiencias pueden involucrar fuertes cambios cognitivoexistenciales y emocionales que deben ser reconocidos; por ejemplo, cuando una persona relata una experiencia anómalo/paranormal, básicamente está buscando una explicación o un significado potencial.

\section{REFERENCIAS}

Alvarado, C. S., \& Zingrone, N. (1999). Out-of-body-experiences among readers of a Spanish New Age magazine. Journal of the Society for Psychical Research, 63. 65-85.

Cardeña, E., Krippner, S., \& Lynn, S.J. (2000). Varieties of anomalous experience: Examining the scientific evidence. Washington. DC: American Psychological Association.

Espinosa Paul, L. (2004). Experiencias aparicionales y absorción psicológica: Un estudio exploratorio on-line. En F. E. Da Silva, F. E. (Ed.). Segun- 
do Encontro Psi: Refletindo Sobre o Futuro da Parapsicologia. Curitiba: Facultades Integradas "Espirita".

Gómez Montanelli, D., \& Parra, A. (2002). Experiencias psi-conflictivas: Una encuesta con implicaciones en parapsicología clínica. Revista Argentina de Psicología Paranormal, 13, 7-47.

Irwin, H., \& Watt, C. (2007). An introduction to parapsychology (5. ${ }^{\mathrm{a}}$ ed.). Jefferson, NC: McFarland.

Kohr, R. (1980). A survey of psi experiences among members of a special population. Journal of the American Society for Psychical Research, 74, 395-412.

Mehrwer Durch Qualitat (2001). Estándares de calidad para la realización de encuestas por internet. Arbeitskreis Deutscher Markt- und Sozialforschungsinstitute [Asociación Alemana de Institutos de Investigación de Mercado y Opinión]. Informe privado. (Traducción: Raúl Páramo Flores).

Parra, A. (2003). Fenómenos paranormales: Una introducción a los eventos sorprendentes. Buenos Aires: Kier.

Parra, A. (2008a). Alucinaciones "negativas": ¿Falla en la percepción o disociación amnésica? Actualidad Psicológica, 33(366), 29-31.

Parra, A. (2008b). Las experiencias extracorpóreas y las experiencias alu- cinatorias: Relación con variables cognitivas y perceptuales. Liberabit, 14, 5-14.

Parra, A. (2008c). Efectos de las experiencias espirituales/paranormales en la vida de las personas y su bienestar psicológico. Revista Argentina de Clínica Psicológica, 17, 233-242.

Parra, A. (2008d). La "visión del aura" como experiencia alucinatoria en individuos no-clínicos. Revista PsicoUSF, 13, 277-286.

Parra, A. (2008e). Medidas psicológicas en relación con experiencias alucinatorias y experiencias aparicionales. Persona, 11, 109-128.

Parra, A. (2009). Experiencias de telepatía teléfonica: Una réplica exploratoria mediante una encuesta on-line. E-Bulletin Psi, 4(3). [Disponible on line en http://www.alipsi.com.ar/eboletin/boletin_psi_4-3_Sept_09. $\mathrm{htm}$.

Parra, A. (2009a). Experiencias alucinatorias nocturnas: Relación con la esquizotipia, tendencias disociativas y propensidad a la fantasía. Revista Internacional de Psicología, 43, 134-143.

Parra, A. (2009b). Variables cognitivas y perceptuales en la experiencia del déjà vu. Acta Psiquiátrica y Psicológica de América Latina, 55(4), 29-36.

Parra, A. (2010b). Experiencias extrasensoriales y experiencias alucina- 
torias: Examinando la hipótesis del continuo de experiencias esquizotípicas. Liberabit, 16(1), 1-10.

Rush, J. H. (1986). Spontaneous psi phenomena: Case studies and field investigations. En Edge, H. L.; Morris, R. L.; Palmer, J. \& Rush, J. (Eds.). Foundation of parapsychology: Exploring the boundaries of human capability (pp. 47-70). Londres: Routledge \& Kegan Paul.

Stokes, D. M. (1997). Spontaneous psi phenomena. En Krippner, S. (Ed.). Advances in parapsychological research 7 (pp. 6-87). Jefferson, NC: McFarland.

Thalbourne, M. A. (1994). The SPR Centenary Census: II. The survey of beliefs and experiences. Journal of the Society for Psychical Research, 59, 420-431.

Thalbourne, M. A. (2001). Relaciones maritales rotas y ocurrencia de fenómenos psi. Revista Argentina de Psicologia Paranormal, 12, 171-175.

Watt, C. (1994). Making the most of spontaneous cases. En Krippner, S. (Ed.). Advances in parapsychological research 7 (pp. 77-103). Jefferson, NC: McFarland.

Zingrone, N. L., \& Alvarado, C. S. (1997). "Broken" marital relations and claims of parapsychological experiences. Trabajo presentado en la $40^{\text {a }}$ Annual Convention of the Parapsychological Association. Brighton, UK. 


\section{Apéndice \\ Experiencias anómalo/paranormales}

\begin{tabular}{|c|c|}
\hline Edad:_______ años & $\begin{array}{l}\text { Sexo: } \\
\text { Masculino } \\
\text { Femenino }\end{array}$ \\
\hline $\begin{array}{l}\text { Estado civil } \\
\text { Soltero } \\
\text { Casado/a } \\
\text { Separada/o } \\
\text { Viudo/a }\end{array}$ & $\begin{array}{l}\text { Orden de nacimiento } \\
\text { Hijo único/a } \\
\text { Primer nacimiento } \\
\text { Segundo nacimiento } \\
\text { Último nacimiento }\end{array}$ \\
\hline $\begin{array}{l}\text { Religión } \\
\text { Católico } \\
\text { Judío } \\
\text { Cristiano no-denominacional } \\
\text { No-denominacional } \\
\text { Filosofías orientales } \\
\text { Agnóstico } \\
\text { Ateo }\end{array}$ & $\begin{array}{l}\text { Espiritualidad } \\
\text { No soy espiritual } \\
\text { Soy un poco espiritual } \\
\text { Soy moderadamente espiritual } \\
\text { Soy muy espiritual } \\
\text { Soy extremadamente espiritual }\end{array}$ \\
\hline
\end{tabular}

\section{Cuestionario}

1. He tenido sueños que anticipaban el futuro

$\square$ Nunca $\quad \square$ Sí, una vez $\quad \square$ Sí, algunas veces $\quad \square$ Sí, casi siempre

Ha sido una experiencia positiva y enriquecedora. $\quad \begin{array}{llllllllll}1 & 2 & 3 & 4 & 5 & 6 & 7 & \text { negativa y perturbadora. }\end{array}$

2. He tenido la experiencia de captar mentalmente el pensamiento de otras personas o transmitir a distancia mis pensamientos.
Nunca
Sí, una vez
Sí, algunas veces
Sí, casi siempre

Ha sido una experiencia positiva y enriquecedora. $\quad \begin{array}{lllllllll}1 & 2 & 3 & 4 & 5 & 6 & 7 & \text { negativa y perturbadora. }\end{array}$

3. He tenido la experiencia de ver campos de energia o luces alrededor del cuerpo de una persona.

$\square$ Nunca $\quad \square$ Sí, una vez $\quad \square$ Sí, algunas veces $\quad \square$ Sí, casi siempre

Ha sido una experiencia

positiva y enriquecedora.

$\begin{array}{lllllll}1 & 2 & 3 & 4 & 5 & 6 & 7\end{array}$

Ha sido una experiencia

negativa y perturbadora. 
4. He tenido la experiencia de sentirme fuera, desprendido o lejos de mi cuerpo físico y observar mi cuerpo a distancia.
Nunca
Sí, una vez
Sí, algunas veces
Sí, casi siempre

Ha sido una experiencia positiva y enriquecedora.

Ha sido una experiencia negativa y perturbadora.

5. A consecuencia de una "muerte clínica" he tenido la experiencia de sentirme fuera de mi cuerpo y regresar con el recuerdo de lo experimentado durante ese trance.
$\square$ Nunca
Sí, una vez
Sí, algunas veces
Sí, casi siempre

Ha sido una experiencia

positiva y enriquecedora.

$\begin{array}{lllllll}1 & 2 & 3 & 4 & 5 & 6 & 7\end{array}$

Ha sido una experiencia

negativa y perturbadora.

6. He experimentado recuerdos que parecen provenir de una vida que he vivido antes de mi nacimiento.

$\square$ Nunca $\square$ Sí, una vez $\square$ Sí, algunas veces $\quad \square$ Sí, casi siempre

Ha sido una experiencia positiva y enriquecedora.

$\begin{array}{lllllll}1 & 2 & 3 & 4 & 5 & 6 & 7\end{array}$

Ha sido una experiencia negativa y perturbadora.

7. Estando solo he tenido la vívida impresión de una sensación de presencia pero no visible donde me encontraba.
Nunca
Sí, una vez
Sí, algunas veces
Sí, casi siempre

Ha sido una experiencia positiva y enriquecedora.

$\begin{array}{lllllll}1 & 2 & 3 & 4 & 5 & 6 & 7\end{array}$

Ha sido una experiencia negativa y perturbadora.

8. He tenido la experiencia de sentir que mi cuerpo no me pertenecía y que estaba siendo controlado por otra fuerza aparte de mí.
Nunca
Sí, una vez
Sí, algunas veces
Sí, casi siempre

Ha sido una experiencia positiva y enriquecedora. negativa y perturbadora.

9. He tenido la experiencia de haber dejado objetos en determinados lugares y cuando voy a buscarlos luego no los encuentro. Tiempo después los encuentro en el mismo lugar donde los dejé, a pesar de haber revisado ese lugar varias veces antes.
Nunca
Sí, una vez
Sí, algunas veces
Sí, casi siempre

Ha sido una experiencia $\begin{array}{lllllllll}\text { positiva y enriquecedora. } & 1 & 2 & 3 & 4 & 5 & 6 & 7 & \text { negativa y perturbadora. }\end{array}$

10. He tenido la experiencia de observar el funcionamiento anormal de bombillas eléctricas, electrodomésticos o computadoras, a veces simultáneamente, de un modo que muy raramente podría ocurrir.
Nunca
Sí, una vez
Sí, algunas veces
Sí, casi siempre

Ha sido una experiencia positiva y enriquecedora. negativa y perturbadora. 
11. He tenido la experiencia de disminuir el dolor de otra persona con solo tocarla.
$\square$ Nunca
Sí, una vez
$\square$ Sí, algunas veces
Sí, casi siempre

Ha sido una experiencia positiva y enriquecedora.

Ha sido una experiencia negativa y perturbadora.

12. He tenido la experiencia de déjà vu, esto es, oír o ver algo que tiene toda la impresión de haberlo oído o visto.
$\square$ Nunca
Sí, una vez
$\square$ Sí, algunas veces
Sí, casi siempre

Ha sido una experiencia positiva y enriquecedora.

Ha sido una experiencia negativa y perturbadora.

13. He tenido una experiencia mística durante un ejercicio de yoga, control mental o meditación.

$\square$ Nunca $\square$ Sí, una vez $\square$ Sí, algunas veces $\quad \square$ Sí, casi siempre

Ha sido una experiencia $\begin{array}{llllllllll}\text { positiva y enriquecedora. } & 1 & 2 & 3 & 4 & 5 & 6 & 7 & \text { negativa y perturbadora. }\end{array}$

14. Estando despierto he tenido la experiencia de oír voces o ver presencias invisibles para otros que me indicaban acerca de un peligro inminente que luego ocurrió.

$\square$ Nunca $\square$ Sí, una vez $\square$ Sí, algunas veces $\quad \square$ Sí, casi siempre

Ha sido una experiencia positiva y enriquecedora. negativa y perturbadora. 